\title{
Smartphone Applications for Mental Health-A Rapid Review
}

Julie Hind, Shannon L. Sibbald

Department of Health Studies, Western University

\section{Abstract}

Objectives: The purpose of this article is to determine what evidence exists about the effectiveness of monitoring and managing mental health via smartphone application. This study aims to inform health care decision makers of available evidence as well as the necessary components and potential barriers to success.

Methods: A rapid review was conducted which yielded eleven primary research studies evaluating nine unique smartphone applications.

Results: The literature identified many benefits of smartphone applications for mental health including: positive perceptions and experiences by patients and doctors as well as their willingness to use the applications to impact their mental health. Further benefits include the apps' ability to provide valid and detailed symptom monitoring, to apply proven psychotherapy methods, to improve access to care and to positively impact clinical outcomes. A potential barrier to success identified by the literature is ensuring patients' long-term adherence to this treatment method. Key success factors to effective monitoring and managing of mental health care via a smartphone app include individually tailored apps, adaptive learning, a feedback system, and clinical, peer and technical support.

Conclusions: Preliminary outcomes in this area seem promising. However, there is a limited number of high-quality research studies and more rigorous scientific investigation into this area is needed.

\section{Background}

The World Health Organization ${ }^{1}$ refers to mental health as "being related to the promotion of well-being, the prevention of mental disorders, and the treatment and rehabilitation of people affected by mental disorders". It is estimated that one in five Canadians are affected each year by mood disorders, anxiety disorders, schizophrenia, attention deficit/hyperactive disorders (ADHD), conduct disorders, oppositional defiant disorders (ODD), substance use disorders, or dementia. ${ }^{2}$ Of the millions of people living with a mental health disorder in Canada, only one in three report that they have sought and received services and treatment. ${ }^{3}$ This will continue to be a huge public health concern predicted to affect more than 8.9 million Canadians by $2041 .^{2}$ Exploring ways to improve the delivery of mental health treatment is of high importance. One opportunity to enhance the delivery of mental health care lies in the use of technology. 4

Smartphones are mobile telephones with computer functionality that allow users to run software applications and connect to the Internet or other data networks. ${ }^{5}$ As of December 2013, there is one smartphone in use for every five people in the world. ${ }^{6}$ The use of applications, or "apps", on smartphones continues to expand with Apple's app store offering more than 850,000 apps as of $2013 .{ }^{7}$ A recent study by Google ${ }^{8}$ found that an average user has 30 apps on their smartphone device with 12 of those apps being used within the past 30 days. Apps offer a graphical user interface, functionality, and usability. ${ }^{13}$ These apps are changing the way people carry out many core activities such as job searching, shopping, and eating out. Although smartphones have made an impact on many aspects of daily living, investigation of the use of smartphone apps to enhance the monitoring and management of mental health has been slow. ${ }^{9}$

Various studies have demonstrated the ability of smartphones to improve mental health, particularly schizophrenia, ${ }^{10,11}$ depression and alcohol dependency, ${ }^{12}$ through the use of short 
message service (SMS) text messaging. A search on the Apple app store in August 2013 revealed more than 4,000 apps with a mental health focus (Table 1). The apps have a variety of uses such as psychoeducation, clinical assessment, resource location, symptom monitoring, tracking treatment progress, skills training, and two-way communication with health care providers. ${ }^{5}$

The purpose of this rapid review is to determine what evidence exists about the effectiveness of monitoring and managing mental health via a smartphone app. Rapid reviews provide an overview of the evidence available regarding a particular topic and to give a sense of the volume and direction of the evidence. ${ }^{14}$ This review aims to inform the discussion around available evidence for managing and monitoring mental health as well as the necessary components and potential barriers to success in making smartphone apps an effective tool to cope with mental health disorders.

\section{Methods}

\section{Rapid Reviews}

Rapid review methodology is typically used to provide evidence to decision makers in health care settings driven by clinical urgency, demands for advances in technology, or limited time and resources. ${ }^{14,15}$ They have been found to provide adequate advice for clinical and policy decisions. ${ }^{16}$

\section{Search Strategy}

Rapid reviews do not employ exhaustive search strategies, but rather use a limited amount of sources in order to expedite the search of the literature. ${ }^{14} \mathrm{~A}$ search was conducted between May 2013 and August 2013 for research articles published in peer-reviewed journals using multiple databases including PubMed, CINAHL, PsychINFO, Scopus, and Web of Science. Search terms included combinations of "mobile health", "mhealth", "smartphone", "mobile phone", "application", "mental health", "depression", "anxiety", "stress", "schizophrenia", "bipolar", "alcohol dependence" and "drug dependence". Hand-searching of exemplar articles was also employed in order to identify additional studies.

\section{Inclusion Criteria}

Abstract review was used to screen all articles retrieved in the original search. An initial focus was placed on locating high-quality systematic reviews. However, this is a relatively new field of research and as such, during the time of this literature search, there was a lack of systematic reviews looking specifically at mental health and the use of apps in managing and monitoring. Consistent with rapid review methodology, primary research articles were included in the absence of systematic reviews. The articles needed to be primary research studies evaluating the efficacy of a smartphone application for monitoring or managing mental health. Studies that focused on computer, personal digital assistants, or SMS texting interventions were excluded. Articles were also excluded if they were not original research articles. No restrictions were placed on geographical location or date of publication. All publications were Englishlanguage articles.

\section{Search Results}

The search yielded eleven primary research studies evaluating nine unique smartphone applications (Table 2). Palmier-Claus et al. ${ }^{17}$ and Ainsworth et al. ${ }^{13}$ evaluated the same clinical trial, as did Reid et al. ${ }^{18}$ and Reid et al. ${ }^{19}$ Three of the studies evaluated a smartphone application targeted at schizophrenia, two targeted youth mental health issues, four targeted stress or depression, one targeted alcohol dependence, and one targeted borderline personality disorder with comorbid substance use disorders. All of the papers were published between 2010 and 2013.

\section{Results}

The results will be discussed in three sections: benefits of mental health apps, key success factors, and barriers to success.
A. Benefits
We identified six benefits of mental health apps in the literature including: positive perceptions and experiences by patients and doctors, patients' willingness to use, valid and detailed symptom monitoring, application of proven psychotherapy methods, positive impact on clinical outcomes, and improvement of access.

\section{Positive Perceptions and Experiences by} Patients and Doctors. A number of studies sought qualitative feedback from patients and doctors. Palmier-Claus et al. ${ }^{17}$ found that individuals with schizophrenia who used a smartphone app to self-report their symptoms perceived the app to be acceptable, valid, and easily integrated into their domestic routines. Patients also reported a positive appeal of usability and functionality. Rizvi, Dimeff, Skutch, 
Carroll, \& Linehan ${ }^{20}$ reported positive perceptions on acceptability and satisfaction in patients with borderline personality disorder and co-morbid substance use disorder. Reid et al. ${ }^{19}$ evaluated a smartphone app used by youth with mental health issues to report their symptoms and have the information communicated to their doctor. The doctors reported an increased understanding of their patients' mental health and found that the app was helpful for informing their decisions about diagnosis, medication, and referrals. ${ }^{19}$

2. Willingness to Use. Two of the studies analyzed the smartphone data to determine patients' usage. McTavish et al. ${ }^{21}$ found that patients with alcohol dependence leaving residential treatment used a smartphone app for ongoing support, resources, and information. In fact, $80 \%$ of patients, including those with comorbid mental illness or drug dependence, were still accessing the application after 16 weeks. Prolonged participation in continuing care is important because it leads to better clinical outcomes. ${ }^{21}$ Ainsworth et al. ${ }^{13}$ compared the usage of a smartphone app to an SMS textingbased system for self-reporting symptoms in patients with schizophrenia. They concluded that the smartphone app was used more frequently and took less time than the text-based system. Although both methods were received positively, patients reported a preference for the smartphone application. This suggests that the success that has been found thus far in engaging patients in SMS-based programs for mental health will likely be just as successful, if not more, if delivered via a smartphone app..$^{10-12}$

3. Valid and Detailed Symptom Monitoring. Palmier-Claus et al. ${ }^{22}$ tested the validity of selfreporting symptoms in patients with schizophrenia. The study compared the data collected by the app with corresponding data collected by conventional, gold standard rating scales (e.g. Positive and Negative Syndrome Scale and Calgary Depression Scale) and found positive symptom scales and affective symptoms showed moderate to strong correlations with the interview measures. These results indicate that the app is a valid method of symptom monitoring. Other studies that used self-monitoring reported improvements in emotional self-awareness. ${ }^{13,23}$ The detailed symptom monitoring was also found as helpful from the doctor's perspective. ${ }^{19} \mathrm{By}$ collecting real-time data, earlier intervention can occur which can help to reduce relapse, selfinjury, and the need for acute care. ${ }^{22}$

4. Apply Proven Psychotherapy Methods. Psychological interventions based on cognitive behavioural therapies (CBT) have proven effective for a range of common mental health disorders. ${ }^{24}$ Many of the studies were able to apply this evidence-based practice to a smartphone app and resulted in improved clinical

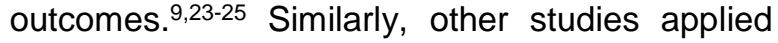
dialectical behaviour therapy and acceptance and commitment therapy. ${ }^{20,24}$

\section{Positive Impact on Clinical Outcomes.} Lappalaine et al. ${ }^{24}$ evaluated the clinical outcomes of patients with mild psychological and stress-related symptoms in a randomized clinical trial. The patients had significant decreases in depressive and psychological symptoms measured with Beck's Depression Inventory and Symptom Checklist-90, respectively, and increases in self-rated health and working ability compared to the control group. However, these outcomes cannot be credited solely to the use of the smartphone app as the intervention group also received treatment via group meetings, a web portal and personal monitoring devices. Nonetheless, $55 \%$ of the treatment group thought the smartphone app was the most useful part of the treatment. These findings suggest that smartphone apps are beneficial when used in combination with other treatment methods. ${ }^{24}$ Harrison et al. ${ }^{9}$ conducted an uncontrolled study in patients with mild-to-moderate stress, anxiety, and depression who used a smartphone app. They found symptoms of stress, anxiety, depression, and overall psychological distress as measured in the Depression and Anxiety Stress Scales were significantly reduced. Also, improvements were found in functional impairment as per the Work and Social Adjustment Scale and perceived self-efficacy for managing one's own mental health as per the Mental Health Self-Efficacy Scale. Watts et al. ${ }^{25}$ tested the results of delivering treatment for major depressive disorder via a smartphone app versus a computer. Both treatment groups experienced positive effects on measures of depression and psychological distress using the Patient Health Questionnaire-9, Beck's Depression Inventory-II and the Anxiety and Depression Checklist K-10 scores. Rizvi et al. ${ }^{20}$ used a smartphone app to treat individuals with borderline personality disorder and comorbid substance use disorders. The study resulted in decreases in emotional intensity and urges to use substances as well as decreases in overall depression symptomatology, as measured by Beck's Depression Inventory. The Brief Symptom Inventory scale also showed a decrease in psychological distress over the course of the trial. 
Reid et al. ${ }^{18}$ did not find significant impacts in health outcomes compared to the attention comparison group in youths with mental health issues who used a smartphone for selfmonitoring. However they did find an increase in emotional self-awareness, consistent with the findings reported by Morris et al. ${ }^{23}$ in individuals suffering from stress.

6. Improved Access. The use of smartphone apps to deliver mental health care has the ability to increase the number of people who have access to treatment. Finally, there are no geographic or socioeconomic barriers. ${ }^{19}$ There are now over 5 billion wireless subscribers with over $70 \%$ of them residing in low and middleincome countries. ${ }^{26}$ There is also a lack of stigma surrounding this delivery method of mental health care, which can help improve the number of patients who seek treatment. ${ }^{23}$ It is particularly well suited for youth (aged 14-24), the age group most commonly affected by mental health issues and unlikely to seek treatment, since mobile phones are their preferred means of communication..$^{9,18}$ Smartphone apps can also broaden the reach by offering support and resources at all times, regardless of location, especially when stressful situations arise. ${ }^{23-24}$

\section{B. Key Success Factors}

Three factors identified by the literature that are critical to the success of the mental health apps are the employment of: (1) individual tailoring and adaptive learning, (2) a feedback system, and (3) clinical, peer, and technical support.

\section{Individual Tailoring and Adaptive Learning.} Individually tailoring the smartphone apps was seen as an important component in many studies. ${ }^{9,13,17,22,24}$ Palmier-Claus et al. ${ }^{22}$ added a degree of personalization to their smartphone app by adding a menu in which the researcher could select which delusions a participants was experiencing therefore customizing the questions they would receive during treatment. By personalizing applications to different needs and preferences of participants, intervention outcomes and adherence may be improved, as they are more likely to see the relevance to their personal care. ${ }^{13,24}$ This aspect is important to patients, as demonstrated by Reid et al., ${ }^{18}$ when concerns were raised about the relevance of the application to their personal situation and the possible threat to the person-centered element of their care. Applications could be improved by employing adaptive learning to tailor questions or to recognize complex patterns and develop algorithms to predict likelihood of relapse. . $^{1,21,23}$ 2. Feedback System. Lappalaine et al. ${ }^{24}$ reported that personal feedback and advice was considered the most useful component of their intervention. Automated or clinician-delivered feedback was suggested as a means to promote acceptance and compliance. ${ }^{13}$ Reid et al. ${ }^{19}$ provided feedback using summary reports with charts, useful resources and recommendations. Adaptive learning could be used to display trends and provide automated feedback. ${ }^{23}$

3. Clinical, Peer and Technical Support. Although this form of treatment is well-received, the human element of care is still highly valued by patients. ${ }^{17,24}$ Smartphone-based assessment should be introduced as an adjunct to, rather than a substitute for, current clinical practice. ${ }^{17}$ Lappalaine et al. ${ }^{24}$ suggested peer and counselor support networks are crucial factors to increase participant engagement and motivation. Technical support is also a key success factor as insufficient instruction was reported as a reason for discontinuation of treatment via app. ${ }^{9} \mathrm{~A}$ high level of assistance and encouragement, especially in the early stages, is important to establish familiarity and increase adherence. ${ }^{17}$

\section{Barriers}

Long-term Adherence. Research has shown that the major challenge associated with delivering mental health treatment via smartphone apps is long-term adherence. A characteristic of many mental health disorders is their chronically relapsing nature, which makes prolonged participation in treatment important in improving clinical outcomes. ${ }^{21}$ However, Ainsworth et al. ${ }^{13}$ reported less than a third of patients with schizophrenia were willing to complete the smartphone assessment for five weeks or longer. Many of the studies were only short-term interventions and some of the longer studies experienced high dropout rates. ${ }^{9}$ Suggestions from the literature on how to encourage long-term adherence and ways to help achieve success with mental health apps are discussed below.

Data Security and Patient Privacy. It is important to consider the data security and patient privacy risks associated with providing apps with personal and health related information. One particular threat is related to unauthorized access or loss of the smartphone. This risk can be reduced through the use of the password-protection feature and through apps that allow smartphones to be wiped remotely, 
such as Apple's Find My iPhone app. Third party encryption apps are also an option to secure data that is stored on the smartphone. Another patient privacy risk is related to an apps' ability to gather and transmit information to software developers or other companies, potentially unencrypted and over insecure network connections. In one study of mobile health and fitness apps, The Privacy Rights Clearinghouse found $40 \%$ of the apps they reviewed were high risk based on the amount of sensitive information they stored and transmitted. ${ }^{27}$ To mitigate this risk, it is recommended that clinicians should be aware of what information apps are collecting and what it is being used for. Patients should then be educated of the privacy risks associated with use of the app in an informed consent procedure. ${ }^{5}$

\section{Discussion \\ Policy Implications}

Smartphone apps have the potential to improve access to mental health treatment and to positively impact clinical outcomes, however more research is needed to understand the best way to implement smartphone apps for mental health. Since consumers are able to browse and purchase apps without many barriers, consideration needs to be given toward what mental health apps ought to be providing and what mental health services need to be monitored by a healthcare provider. Given the increased availability and use of smartphone apps, consideration should be given for smartphone usage policies, policies on education by healthcare providers (and who within a healthcare team should provide the education) as well as associated policies to support smartphone accessibility.

Mental health apps are often utilized by the consumer without medical supervision, although this is not always the case. Assuming that these apps offer some benefit to a broader treatment regime, there may be value in exploring the cost-effectiveness of such apps. Cost was not discussed as a barrier since smartphones and apps were provided free of charge in the studies. If users are to bear any of the costs of the smartphone device, the data charges, or the app, then cost itself may become a barrier to this method of treatment.

App creators need to consider the implications of dealing in such a sensitive area and be aware of the potential challenges. There is no current structure for accountability or responsibility once a patient has a mental health app-which could foreseeably lead to other challenges. A new trend can be seen with some health care providers actually prescribing apps to patients as a part of a larger treatment plan. ${ }^{28}$

\section{Limitations and Future Directions}

The use of rapid review methodology may introduce selection bias, publication bias and language of publication bias, as search strategies were not comprehensive. Another limitation of this review is the quality of studies included. Due to limited research available, only five of the studies included were randomized control trials. ${ }^{16,18,19,21,24}$ Variability in sample size, design, and data collection methods employed in each of the reviewed studies leads to difficulty in comparing studies. In the future, more randomized control trials should evaluate the effectiveness of mental health applications. Furthermore, researchers should begin to study the effects of more advanced applications with individual tailoring and adaptive learning.

\section{Conclusion}

While a copious amount of mental health apps are available, there is a scarce amount of evidence-based, evaluated apps. ${ }^{25}$ Although there is a limited amount of high-quality research studies, preliminary outcomes seem promising. Considering the ubiquity of smartphone apps, the positive perceptions of patients and doctors, and their potential benefits, more rigorous scientific investigation into this area is needed. ${ }^{12}$

\section{References}

1. World Health Organization. Mental Health. 2013. Available from: http://www.who.int/topics/mental_health/en

2. Smetanin, P., Stiff, D., Briante, C., Adair, C., Ahmad, S., Khan, M. The life and economic impact of major mental illnesses in Canada: 2011 to 2041. RiskAnalytica, on behalf of the Mental Health Commission of Canada. Dec 2011. Available from: www.mentalhealthcommission.ca/English/s ystem/files/private/document/MHCC_Repo rt_Base_Case_FINAL_ENG_0.pdf

3. Statistics Canada. Canadian community health survey: Mental health and wellbeing. The Daily. Sept 2003. Available from: http://www.statcan.gc.ca/daily-quotidien/030903/dq030903a-eng.htm

4. Ehrenreich, B., Righter, B., Rocke, D. A., Dixon, L., Himelhoch, S. Are mobile phones and handheld computers being 
used to enhance delivery of psychiatric treatment? A systematic review. Journal of Nervous and Mental Disease. 2011;199(11):886-891.

5. Luxton, D. D., McCann, R. A., Nigel, B. E., Mishkind, M. K., Reger, G. M. mHealth for Mental Health: Integrating Smartphone Technology in Behavioural Healthcare. Professional Psychology: Research and Practice. 2011;42(6):505-512.

6. Business Insider. 1.5 Billion Smartphones In The World. Feb 2013. Available from: http://www.businessinsider.com/15-billionsmartphones-in-the-world-22013-2

7. Apple. Apple Press Info- Apple's App Store Marks Historic 50 Billionth Download. May 2013. Available from: http://www.apple.com/pr/library/2013/05/16Apples-App-StoreMarks-Historic-50-Billionth-Download.html

8. Google. Our Mobile Planet: Canada. Understanding the Mobile Consumer. May 2013. Available from: http://services.google.com/fh/files/misc/omp-2013-ca-en.pdf

9. Harrison, V., Proudfoot, J., Wee, P. P., Parker, G.,Pavlovic, D. H., Manicavasagar, V. Mobile mental health: Review of the emerging field and proof of concept study. Journal of Mental Health. 2011;20(6):509524.

10. Spaniel, F., Vohlídka, P., Kozený, J., Novák, T., Hrdlicka, J., Motlová, L., Cermak, J,, Hoschl, C. The Information Technology Aided Relapse Prevention Programme in Schizophrenia: an extension of a mirror-design follow-up. Int $\mathrm{J}$ Clin Pract. 2008;62(12):1943-1946.

11. Granholm, E., Ben-Zeev, D., Link, P. C., Bradshaw, K.R., Holden, J.L. Mobile Assessment and Treatment for Schizophrenia (MATS): a pilot trial of an interactive text-messaging intervention for medication adherence, socialization, and auditory hallucinations. Schizophr Bull. 2012;38(3):414-425.

12. Agyapoing, V. I. O., Ahern, S., McLoughlin, D. M., Farren, C. K. Supportive text messaging for depression and comorbid alcohol use disorder: Single-blind randomized trial. Journal of Affective Disorders. 2012;141(2-3):168-176.

13. Ainsworth, J., Palmier-Claus, J. E., Machin, M., Barrowclough, C., Dunn, G., Rogers, A., Buchan, I., Barkus, E., Kapur, S., Wkyes, T., Hopkins, R. S., Lewis, S. A Comparison of Two Delivery Modalities of a Mobile Phone-Based Assessment for
Serious Mental Illness: Native Smartphone Application vs Text-Messaging Only Implementations. Journal of Medical Internet Research. 2013;15(4):e60.

14. Khangura, S., Konnyu, K., Cushman, R., Grimshaw, J., Moher, D. Evidence summaries: the evolution of a rapid review approach. Systematic Reviews. 2012;1(10).

15. Ganann, R., Ciliska, D., Thomas, H. Expediting systematic reviews: methods and implications of rapid reviews. Implementation Science. 2010;5(56).

16. Watt, A., Cameron, A., Sturm, L., Lathlean, T., Babidge, W., Blamey, S., Facey, L., Hailey, D., Norderhaug, I., Maddern, G. Rapid versus full systematic reviews: validity in clinical practice? ANZ J Surg. 2008;78:1037-1040.

17. Palmier-Claus, J. E., Rogers, A., Ainsworth, J., Machin, M., Barrowclough, C., Laverty, L., Barkus, E., Kapur, S., Wykes, T., Lewis, S. W. Integrating mobilephone based assessment for psychosis into people's everyday lives and clinical care: a qualitative study. BMC Psychiatry. 2013;13(34).

18. Reid, S. C., Kauer, S. D., Hearps, S. J. C., Crooke, A. H. D., Khor, A. S., Sanci, L. A., Patton, G. C. A mobile phone application for the assessment and management of youth mental health problems in primary care: a randomized controlled trial. BMC Family Practice. 2011;12(131).

19. Reid, S. C., Kauer, S. D., Hearps, S. J. C., Crooke, A. H. D., Khor, A. S., Sanci, L. A., Patton, G. C. A mobile phone application for the assessment and management of youth mental health problems in primary care: health service outcomes from a randomized controlled trial of mobiletype. BMC Family Practice. 2013;14(84).

20. Rizvi, S. L., Dimeff, L. A., Skutch, J., Carroll, D., Linehan, M. M. A Pilot Study of the DBT Coach: An Interactive Mobile Phone Application for Individuals With Borderline Personality Disorder and Substance Use Disorder. Behavior Therapy. 2011;42(4):547-762.

21. McTavish , F. M., Chih, M., Shah, D., Gustafson, D. H. How Patients Recovering From Alcoholism Use a Smartphone Intervention. Journal of Dual Diagnosis. 2012;8(4):294-304.

22. Palmier-Claus, J. E., Ainsworth, J., Machin, M., Barrowclough, C., Dunn, G., Barkus, E., Rogers, A., Wykes, T., Kapurm 
S., Buchan, I., Salter, E., Lewis, S. W. The feasibility and validity of ambulatory selfreport of psychotic symptoms using a smartphone software application. BMC Psychiatry. 2012;12(172).

23. Morris, M. E., Kathawala, Q., Leen, T. K., Gorenstein, E. E., Guilak, F., Labhard, M., Deleeuw, W. Mobile Therapy: Case Study Evaluations of a Cell Phone Application for Emotional Self-Awareness. Journal of Medical Internet Research. 2010:12(2);e10.

24. Lappalainen, P., Kaipainen, K., Lappalainen, R., Hoffrén, H., Myllymäki, T., Kinnunen, M., Mattila, E., Happonen, A. P., Rusko, H., Korhonen, I. Feasilbility of a Personal Health Technology-Based Psychological Intervention for Men with Stress and Mood Problems: Randomized Controlled Pilot. JMIR Res Protoc. 2013;2(1).

25. Watts, S., Mackenzie, A., Thomas, C., Griskaitis, A., Mewton, L., Williams, A.,
Andrews, G. CBT for depression: a pilot RCT comparing mobile phone vs. computer. BMC Psychiatry. 2013;13(49).

26. World Health Organization. mHealth: New horizons or health through mobile technologies. 2011. Available from: http://www.who.int/goe/publications/goe_mhealth_w eb.pdf

27. Privacy Rights Clearinghouse. Mobile Health and Fitness Apps: What Are the Privacy Risks. July 2013. Available from: https://www.privacyrights.org/fs/fs39mobile-health-and-fitness-apps-what-areprivacy-risks

28. Lippman, H. How Apps Are Changing Family Medicine: Clinicians Reviews. Clinician Reviews. 2013;23(9):26-31. Available from: http://www.clinicianreviews.com/home/article/how-apps-arechanging-family-medicine.html. 
Table 1- Examples of Mental Health Applications

\begin{tabular}{|c|c|c|c|}
\hline Topic & Keyword search & Number of Apps & Examples \\
\hline Mood Disorders & $\begin{array}{l}\text { Mood, stress, } \\
\text { depression, bipolar }\end{array}$ & 3307 & $\begin{array}{l}\text { iMoodJournal, MoodKit-Mood } \\
\text { Improvement Tools, Deal with } \\
\text { Depression, Stress Check }\end{array}$ \\
\hline Anxiety Disorders & Anxiety, phobia & 650 & $\begin{array}{l}\text { Panic Attacks? Learn how to deal } \\
\text { with panic attacks, Anxiety Free, } \\
\text { Manage Anxiety }\end{array}$ \\
\hline Psychotic & Schizophrenia & 17 & $\begin{array}{l}\text { Psychiatry pocket, MedAid24x7, } \\
\text { iBiomed }\end{array}$ \\
\hline $\begin{array}{l}\text { Childhood and } \\
\text { Adolescence } \\
\text { Disorders }\end{array}$ & ADHD, ODD & 203 & ADHD Tracker, ADHD Treatment \\
\hline $\begin{array}{l}\text { Cognitive } \\
\text { Impairment }\end{array}$ & $\begin{array}{l}\text { Alzheimer's, } \\
\text { dementia }\end{array}$ & 187 & $\begin{array}{l}\text { Dementiadge, ALZTrack, Memory } \\
\text { Manager }\end{array}$ \\
\hline $\begin{array}{l}\text { Substance Use } \\
\text { Disorders }\end{array}$ & $\begin{array}{l}\text { Alcohol abuse, drug } \\
\text { abuse }\end{array}$ & 34 & $\begin{array}{l}\text { RecoveryNow, Sober Day, } \\
\text { Overcoming Alcoholism }\end{array}$ \\
\hline
\end{tabular}

NOTE: Applications may fall under multiple categories. 
Table 2 - Included Studies

\begin{tabular}{|c|c|c|c|c|c|c|c|c|c|c|c|}
\hline 节 & 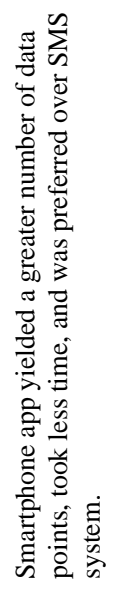 & 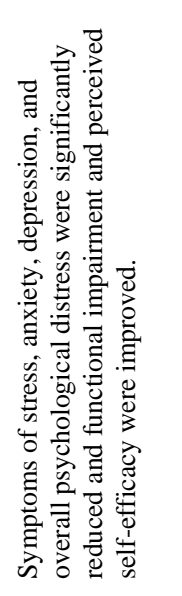 & 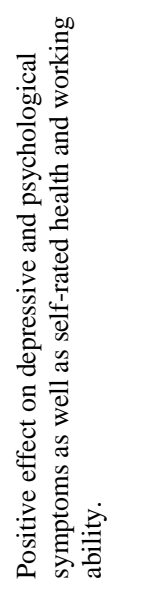 & 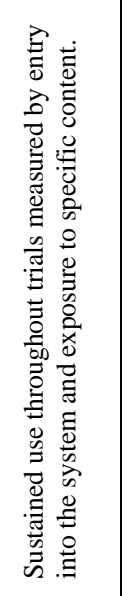 & 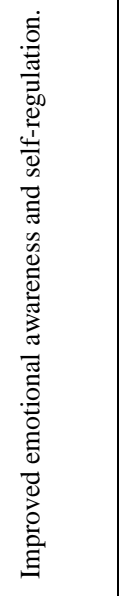 & 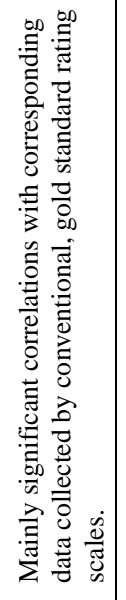 & 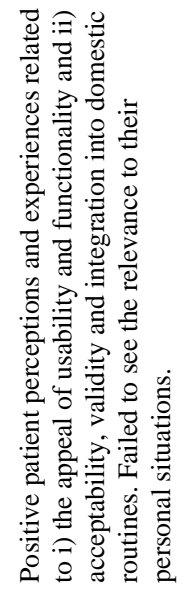 & 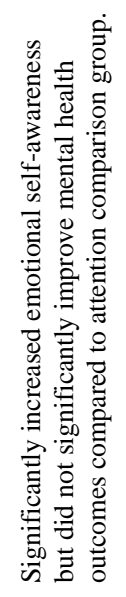 & 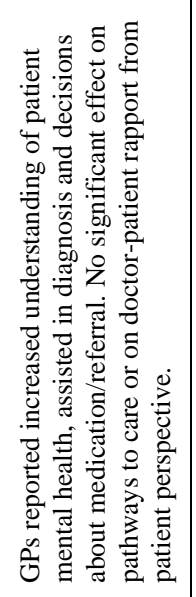 & 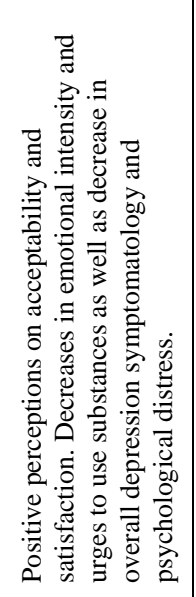 & 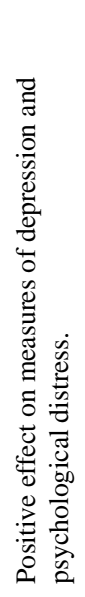 \\
\hline 氞 & 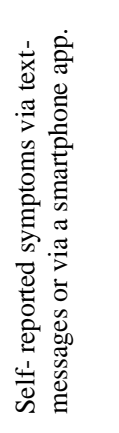 & 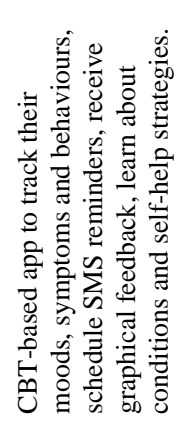 & 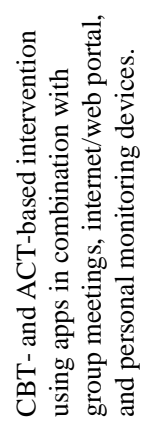 & 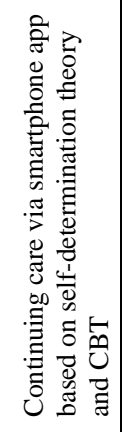 & 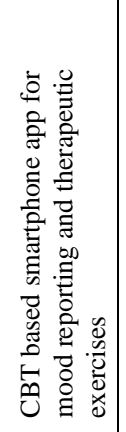 & 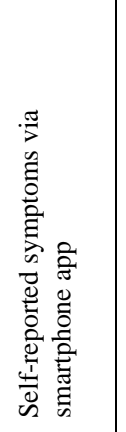 & 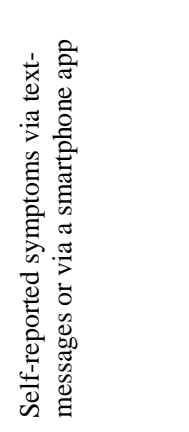 & 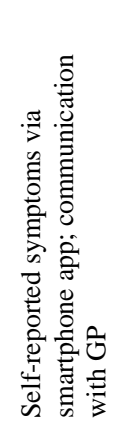 & 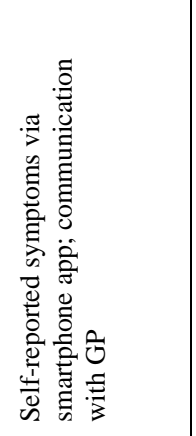 & 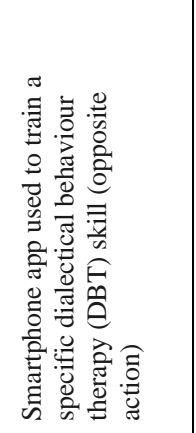 & 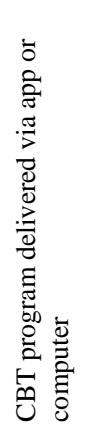 \\
\hline 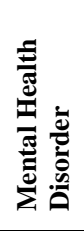 & 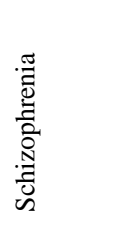 & 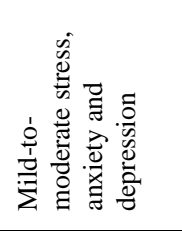 & 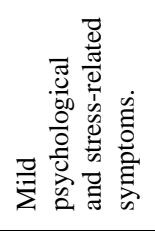 & 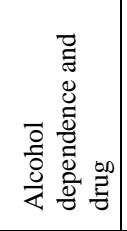 & 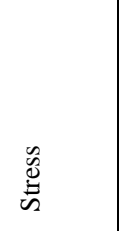 & 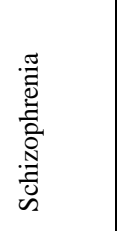 & 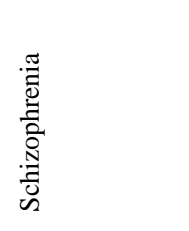 & 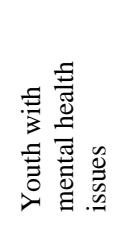 & 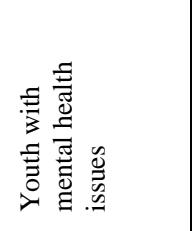 & 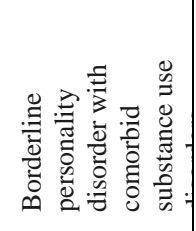 & 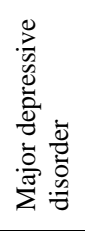 \\
\hline 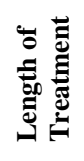 & 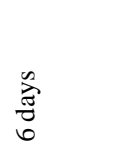 & 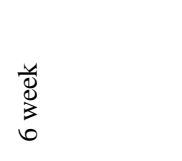 & 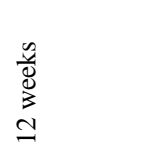 & 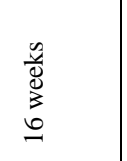 & $\begin{array}{l}n \\
\frac{n}{0} \\
3 \\
+\end{array}$ & $\begin{array}{l}\frac{y}{0} \\
\frac{0}{3} \\
-\end{array}$ & 兽 & 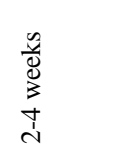 & 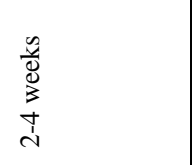 & 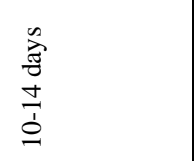 & $\begin{array}{l}\tilde{v} \\
\mathbb{0} \\
\vdots \\
\infty\end{array}$ \\
\hline 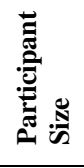 & $\stackrel{\sim}{\sim}$ & $\forall$ & $\stackrel{\sim}{\sim}$ & 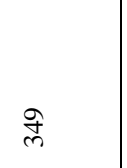 & $\infty$ & ల & $\stackrel{\sim}{\sim}$ & $\stackrel{\Xi}{\Xi}$ & $\stackrel{\Xi}{\Xi}$ & $\tilde{\mathrm{N}}$ & $\ddot{m}$ \\
\hline 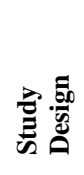 & 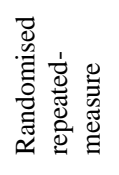 & 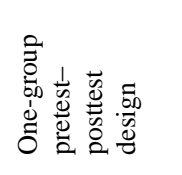 & 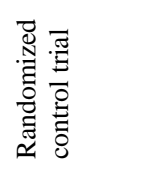 & 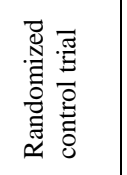 & 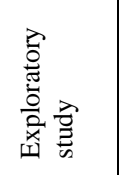 & 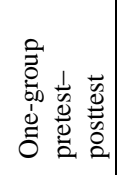 & 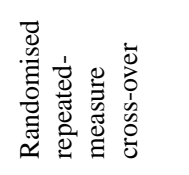 & 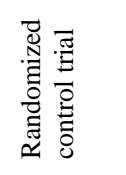 & 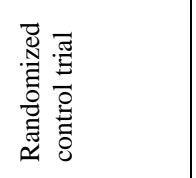 & 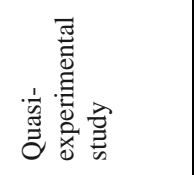 & 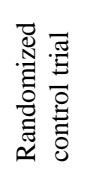 \\
\hline 鄫 & 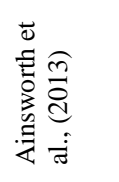 & 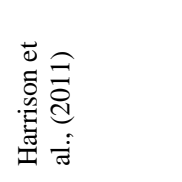 & 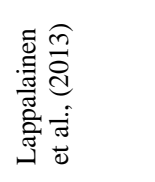 & 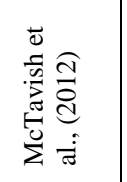 & 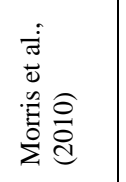 & 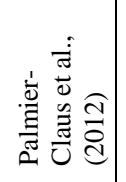 & 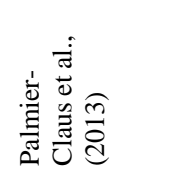 & 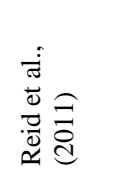 & 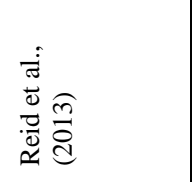 & 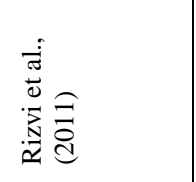 & 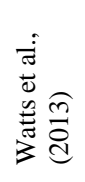 \\
\hline
\end{tabular}

